\title{
Mainstream Consensus and the Expansive Fringe: Characterizing the Polarized Information Ecosystems of Online Climate Change Discourse
}

\author{
Joshua Uyheng \\ CASOS, Inst. for Software Research \\ Carnegie Mellon University \\ Pittsburgh, Pennsylvania, USA \\ juyheng@cs.cmu.edu
}

\author{
Aman Tyagi \\ CASOS, Engineering \& Public Policy \\ Carnegie Mellon University \\ Pittsburgh, Pennsylvania, USA \\ amant@cs.cmu.edu
}

\author{
Kathleen M. Carley \\ CASOS, Inst. for Software Research \\ Carnegie Mellon University \\ Pittsburgh, Pennsylvania, USA \\ kathleen.carley@cs.cmu.edu
}

\begin{abstract}
In this paper, we introduce a social network perspective for characterizing polarized information ecosystems. We apply our framework to a large-scale dataset of climate change conversations on Twitter. Leveraging a stance detection algorithm, we quantify the linksharing behaviors of Believers and Disbelievers of anthropogenic climate change. We generate networks of web domains based on co-sharing by Believers and Disbelievers, and we characterize these networks in terms of both structure and content. While Believers outnumber Disbelievers in our dataset, our results showed that Disbelievers are responsible for sharing over three times as many unique domains. However, for every $10 \%$ increment in a domain's proportion of Disbeliever sharers, the same domain is shared $58 \%$ less. Structurally, we observed that domain clusters associated with Believers were significantly smaller, denser, and less connected to other domains. Content-wise, we additionally found that Disbelievers were the near-exclusive sharers of majority right-wing and known fake news sources, whereas both Believers and Disbelievers shared left-wing and centrist domains. Collectively, our findings indicate that climate change Believers rely on a popular, well-consolidated, and exclusive set of mainstream web domains. In contrast, Disbelievers draw on a diverse and fragmented collection of fringe information sources. Although these results suggest the marginal status of climate change skepticism, they also troublingly point to its expansive ecosystem of online information featuring multiple entry points. We conclude with directions for future research and potential implications for science communication and climate change policymakers.
\end{abstract}

\section{CCS CONCEPTS}

- Applied computing $\rightarrow$ Sociology; Environmental sciences; • Information systems $\rightarrow$ Web mining; • Computing methodologies $\rightarrow$ Machine learning.

This work is licensed under a Creative Commons Attribution International 4.0 License.

WebSci '21, June 21-25, 2021, Virtual Event, United Kingdom

(C) 2021 Copyright held by the owner/author(s)

ACM ISBN 978-1-4503-8330-1/21/06.

https://doi.org/10.1145/3447535.3462486

\section{KEYWORDS}

climate change, polarization, information ecosystems, social network analysis

ACM Reference Format:

Joshua Uyheng, Aman Tyagi, and Kathleen M. Carley. 2021. Mainstream Consensus and the Expansive Fringe: Characterizing the Polarized Information Ecosystems of Online Climate Change Discourse. In 13th ACM Web Science Conference 2021 (WebSci '21), fune 21-25, 2021, Virtual Event, United Kingdom. ACM, New York, NY, USA, 9 pages. https://doi.org/10.1145/3447535.3462486

\section{INTRODUCTION}

Public discourse surrounding climate change has chronically been characterized by a state of polarization [7, 12, 25, 26]. Such dynamics have been studied in both real-world and digital contexts, featuring conflicts between those who accept the scientific reality of anthropogenic climate change, and those who reject it $[9,19]$. In particular, researchers across various disciplines have uncovered significant insights into how polarized discourse is linked with social and emotional rifts, leading to the formation of echo chambers, exacerbating hostile interactions, and the spread of climate misinformation $[1,18,29]$.

Yet especially relevant to cyberspace is the embeddedness of online conflicts within informational divides [17]. Varying information that is available to different groups may shape different understandings of reality $[4,5]$. One significant way of sharing information on social media is via sharing links (or URLs). These links could form information ecosystems driven by homophily in the way users interact on social media websites. These ecosystems could exacerbate divisions between communities, further leading to extreme viewpoints [22].

This paper examines how such information ecosystems may shape the public conversation in climate change discussion. First, we classify users into climate change belief groups, users who believe in the anthropogenic cause of climate change (Believers), and users who do not (Disbelievers). Second, we compare and contrast the link-sharing pattern of both groups. Last, we explore the structure and content of these information ecosystems. More specifically, in this paper, we answer the following research questions:

(1) What link-sharing (or URL-sharing) behaviors do accounts with different stances on climate change engage in?

(2) How can we characterize the structure of information ecosystems around online climate change discourse?

(3) How can we characterize the content of information ecosystems around online climate change discourse? 
To answer these research questions, we utilize a combination of machine learning and network science tools to computationally examine a large-scale, long-term dataset of Twitter conversations about climate change [27]. By examining the network dynamics of link-sharing (URL-sharing) practices by various groups holding different stances toward climate change, we unearth novel characterizations of the distinct information ecosystems that drive polarized discourse around climate change. We specifically triangulate evidence of polarized bifurcation between a consolidated, mainstream information ecosystem that bolsters the scientific consensus around climate change; and an expansive, fringe information ecosystem that may support diverse entry points into climate change skepticism $[1,29]$. These findings resonate with and extend recent scholarship on information polarization around climate change and other controversial topics of public discourse [2, 30, 31, 33].

To lay the groundwork for this paper and its contributions, Section 2 presents an overview of related work around the key concepts of information ecosystems, polarization, and climate change discourse. Section 3 then provides more detail about our dataset and proposed methodology. Finally, we present our findings in Section 4 and conclude with their implications and directions for future work in Section 5.

\section{RELATED WORK}

\subsection{Information Ecosystems and Online Discourse}

Online social networks facilitate interactions and information exchange between large publics. However, due to various psychological, social, and technological factors, social media platforms have become less like avenues for consensus and open discourse [6], and instead become riddled with silos of opposing bodies of information and similarly divided communities which subscribe to them [8]. Individuals are motivated to seek out information that affirms existing beliefs; they are also more likely to share these affirmative sources of knowledge with others holding adjacent views [28, 32]. This reciprocal relationship between affinities for affirmative information and like-minded others contributes to an informational basis for group polarization [17].

In this work, we refer to information ecosystems as these interconnected sources of knowledge forming the basis of online communities. Research abounds with evidence that conflicting sources of information correspondingly lead to not just differing views of reality [10], but also increased animosity against others holding distinct beliefs [5]. However, past work also indicates nuance to this broad narrative of polarized information ecosystems. Some studies, for instance, suggest that echo chamber effects may be overstated, and that most consumers of online information engage with diverse information sources [2]. Other research shows that these effects are asymmetrical [13]. Whereas some groups might be motivated to consume more homogeneous sources of information for various reasons, other groups may tend to accept information from more diverse sources and of varying levels of credibility [33]. Still, more research points out that even if social divides are not extreme to begin with, divided information ecosystems may nonetheless reinforce perceptions of polarization, which subsequently generate hostility in a self-fulfilling cycle [31].

\subsection{Polarization and Climate Change}

These issues are particularly salient in the context of online climate change discourse. The problem of anthropogenic climate change has constituted a significant source of conflict both online and in the real-world $[9,12]$. Information ecosystems in this area have been well-studied with respect to news coverage and framing by media institutions. Scholars report that a highly diverse media environment characterizes the public's access to information about climate change, thereby leading to various forms of polarization in public discourse about the topic [4, 7].

The enormity of climate change as a planetary phenomenon, social scientists argue, leads to a desire for cognitive closure [18]. Because of the similarly vast scale of climate change information online, diverse digital populations may indeed be drawn to scientific knowledge; yet at the same time, these motivations may also drive consumption of non-traditional alternatives which promise epistemic certainty [29]. Traditional media institutions, climate change activists, as well as motivated skeptics employ the resources of cyberspace with both proficiency and influence $[1,30]$. It is therefore important to understand not just extant divisions in the kinds of information available to online discourse, but also how these resources are employed by different online communities.

In this view, prior scholarship has similarly attempted to analyze digital publics' online behaviors around climate change. For instance, work by Pearce et al. [21] analyzed popular topics among Twitter accounts who were supportive, unsupportive, or neutral to the 2013 Intergovernmental Panel on Climate Change's (IPCC) Fifth Assessment Report (AR5). Tyagi et al. [25] showed the existence of echo chambers around the IPCC's 24th Conference of Parties (COP). Asymmetric polarization effects were also found by Tyagi et al. [26] with respect to the level of affective polarization between online groups, finding that skeptics of anthropogenic climate change tended to be more hostile on Twitter. Previous studies have also shown that when polarized communities communicate with each other, conversations related to the dismissal of climate change as a hoax and higher incivility levels prevail [3].

\subsection{Contributions of This Work}

In this paper, we bridge these concerns with polarization, information ecosystems, and online climate change discourse through the use of a computational methodology. While relying on fundamental analytical techniques, our methodology enforces a novel theoretical understanding of information ecosystems through the lens of social networks. This network-based perspective facilitates the derivation of key empirical insights regarding the collective information-sharing practices of climate change Believers and Disbelievers. In sum, our contributions are as follows:

(1) we extend existing work on various aspects of polarization around media representations of climate change to account for the large-scale behaviors of online communities;

(2) we introduce a computational framework for characterizing polarized information ecosystems; and

(3) we identify consolidated information ecosystems around mainstream consensus about the reality of anthropogenic climate change, versus more diffuse yet expansive information ecosystems of skepticism and misinformation. 


\section{DATA AND METHODS}

\subsection{Dataset}

We collected a large dataset of climate change tweets from August 26, 2017 to September 14, 2019. This dataset represented over 38 million unique tweets and retweets from 7 million unique accounts. Using Twitter's standard API ${ }^{1}$, we used the following keywords as search parameters to capture prevailing indicators of online climate change discussion: "Climate Change", "\#ActOnClimate", "\#ClimateChange" in this time period. Data collection was briefly paused twice, and so analysis omits these time periods: April 7 May 21, 2018, and May 12 - 16, 2019. To facilitate temporal analysis, data were aggregated on a monthly basis, beginning with the 26th of each month. This resulted in a total of 25 monthly snapshots of the online climate change discussion.

\subsection{Stance Detection}

Acknowledging the diversity of stances toward climate change, we rely here on a binary heuristic that distinguishes between climate change 'Believers' - or those who accept the reality of anthropogenic climate change - and climate change 'Disbelievers' - or those who reject it. Though this does not capture the complexity of real-world beliefs, this framework allows for the tractability of our analysis, and nonetheless captures the broadest differences between various communities of belief and skepticism in line with our interest in polarization $[25,26]$. To classify users according to this heuristic taxonomy, we employ a stance detection procedure that associates each user with a label of climate change Believer, or Disbeliever [16] Numerically, Believers are assigned a score of +1 , and Disbelievers a score of -1 .

Our framework uses a weak supervision approach with cotraining and label propagation [16]. First, we provide a set of seed hashtags which are consistently used only by Believers or only by Disbelievers. Confident labels are then assigned to accounts that use these hashtags. Next, a supervised machine learning text classifier is trained with these known instances to assign labels to other accounts in our dataset. Labels are additionally propagated using user-retweet and user-hashtag networks, where it is assumed that users tend to retweet others of the same stance. Finally, this framework repeats this process to iteratively label accounts based on increasingly confident predictions about user stance. Prior research has successfully adopted this approach in climate change research $[24,26]$.

For this work, we used the hashtags \#ClimateChangeIsReal and \#SavetheEarth to set seeds for climate change Believers. For Disbelievers, we used \#ClimateHoax and \#Qanon. These hashtags have been shown to be used consistently by their respective stance groups [25] ${ }^{2}$. This method divides our corpus of unique users into 3.9 million Believers and 3.1 million Disbelievers. We then manually evaluate the labels of 2,000 randomly chosen users to find $\sim 81 \%$ classification accuracy of Believers and Disbelievers.

\footnotetext{
${ }^{1}$ https://developer.twitter.com/en/docs/tweets/search/overview/standard

${ }^{2}$ We tested belief classification using the following hashtags to find similar results Disbeliever hashtags: ClimateHoax, YellowVests and Qanon. Believer hashtags: ClimateChangeIsReal,ClimateActionNow, FactsMatter, ScienceMatters, ScienceIsReal
}

\subsection{Social Network Analysis}

3.3.1 Network Representation. To examine the structure and content of information ecosystems, we use a social network approach to represent the evolving online conversation. First, we truncate each individual link to its domain. This means all links to the same website are represented as the same entity. Next, for the month $m$ out of $M$ months, let $G_{m}$ be the bipartite account-to-domain network, where edges are weighted by the number of times a given account shares links from a given domain within the month under consideration. We generate one such network for each month. Then, we obtain the folded, unimodal domain-by-domain network $H_{m}=G_{m}^{T} G_{m}$, which captures the co-sharing of web domains by the same accounts.

To each domain $D$ among the vertices in $H_{m}$, we also assign a stance score, utilizing the stance scores of accounts sharing that domain within month $m$. In particular, let $X_{m}(D)$ be the set of all accounts that share $D$ within month $m$, and let $s(x)$ represent the stance of each $x \in X_{m}(D)$. Then the corresponding domain-level stance $s_{m}(D)$ is given by Equation 1:

$$
s_{m}(D)=\frac{1}{\left|X_{m}(D)\right|} \sum_{x \in X_{m}(D)} s(x) .
$$

We note that, since online accounts discussing issues related to climate change may also mention unrelated topics, we may also find several domains which ostensibly do not focus on climate change. Hence, a stance score assigned to a given domain does not necessarily mean that the domain itself holds a particular stance in relation to climate change. But it may reflect broad imbalances in whether Believers or Disbelievers in our datasets are more likely to share links to these websites.

3.3.2 Community Detection. For much of the analysis in this work, we further take clusters within these domain-by-domain networks. These domain clusters represent groups of domains that tend to be shared by the same accounts, thus operationalizing our conceptualization of information ecosystems. We apply Leiden clustering to these domain-by-domain networks, a state-of-the-art community detection algorithm on unimodal networks, to generate these cluster assignments [23].

3.3.3 Structural Features of Domain Clusters. Finally, using these domain clusters, we zero in on particular structural features to characterize the information ecosystems of Believers and Disbelievers. More specifically, we examine the size of these clusters, which refers to the number of domains assigned to the given cluster; the density of these clusters, a classical network science measure that refers to the fraction of possible in-cluster edges which are realized; and the E/I index, a measure which captures how a given cluster is isolated from (negative values) versus integrated with (positive values) other clusters in the network [15].

\subsection{Quantitative Content Analysis}

3.4.1 Political Orientation of Web Domains. We define the rightleaning, left-leaning, and center-leaning audience news media as per Pew Research Center's study [14]. The study relied on a survey of a large population in the USA to find popular news media's political orientation. We manually found the corresponding primary 
domains of these news agencies' websites for each of the news media agencies reported in the study. These domains are reported in the Appendix. Echoing Young and Anderson [33], we were interested to see whether the partisanship of information sources varied with respect to climate change stance. For a given domain cluster $D$, we classify it as majority left, majority right, or majority center based on its constituent domains.

3.4.2 Web Sources of Fake News. To examine the prevalence of misinformation in our dataset, we use a corpus of fake news sites by Huang and Carley [11]. Motivated by prior work on the prevalence of low-credibility information around the online climate change discourse [18], we examine whether each domain cluster $D$ contains a known fake news domain with reference to this corpus. Notably, a list-based approach like ours may be unable to detect novel misinformation sources, but they nonetheless capture an important signal regarding more well-established sources of low-credibility information.

3.4.3 Identifying Central Domains. Finally, to zoom in on particular noteworthy domains in our dataset, we focused on the most persistently central domains over time. For each month $m$, each domain $D$ is associated with a cluster-level centrality measure $C_{m}(D)$, which is the sum of the weights of all edges connecting $D$ with other domains in the cluster to which $D$ is assigned. We specifically chose this cluster-level centrality measure instead of more global ones since the latter are biased solely toward domains which receive the most shares overall. Cluster-level centrality, on the other hand, allows for greater variation among globally popular websites, as well as those more concentrated among a specific group of accounts. We then focus on the most central accounts for both Believers and Disbelievers, that is, those which respectively obtain the highest values of $C_{m}^{b}(D)$ and $C_{m}^{d}(D)$. These are computed according to Equations 2 and 3 :

$$
\begin{gathered}
C_{m}^{b}(D)=\frac{1}{M} \sum_{m=1}^{M}\left[C_{m}(D) \times \operatorname{sign}\left(s_{m}(D)\right)\right] \\
C_{m}^{d}(D)=-\frac{1}{M} \sum_{m=1}^{M}\left[C_{m}(D) \times \operatorname{sign}\left(s_{m}(D)\right)\right] .
\end{gathered}
$$

\section{RESULTS}

Our findings revealed evidence of distinct link-sharing behaviors between climate change Believers and Disbelievers. As we show below, these results further point to unique features of their respective information ecosystems. The subsequent sections organize our analysis into three core questions, tackling differences in individual link-sharing behaviors, characterizing structural features of online climate change information ecosystems, and mapping the distinctive content of these information ecosystems.

\subsection{How much link-sharing (or URL-sharing) do climate change Believers and Disbelievers engage in?}

First, we examine individual link-sharing behaviors. Figure 1 depicts the relative level of link-sharing engaged in by Believers and Disbelievers, compared to the relative presence of Believer and
Disbeliever accounts in the dataset. Here, we observe a distinct imbalance: although Believers outnumber Disbelievers in terms of accounts participating in online climate change discourse, Disbelievers engage in a much larger proportion of link-sharing.

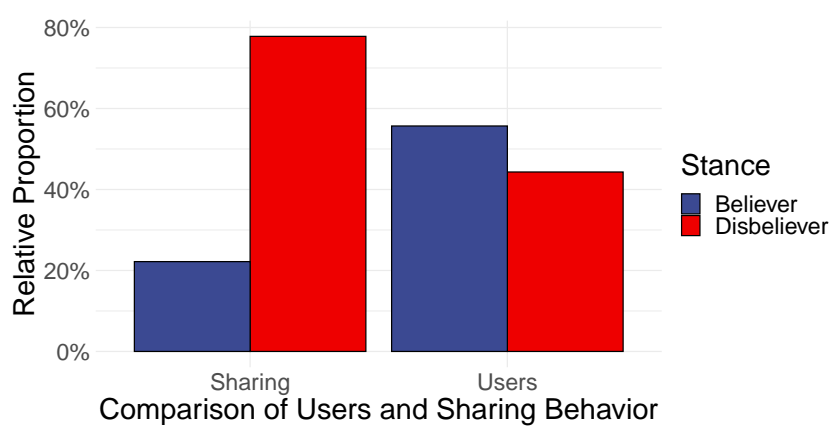

Figure 1: Comparison of relative link-sharing behavior versus presence in dataset of climate change Believers and Disbelievers.

In particular, as seen above, Disbelievers produce $77.81 \%$ of the link-sharing in our dataset, whereas Believers comprise the remaining 22.19\%. This 7-to-2 ratio shows a sharp contrast to Disbelievers making up $44.32 \%$ of the accounts in our dataset, with Believers being the remaining $55.68 \%$ - a 4-to-5 ratio. A two-sample test for equality of proportions quantifies this disparity, confirming that these two distributions are far from equal $\left(\chi^{2}=2518418, p<.001\right)$. In other words, while there may be more Believers in the conversation, Disbelievers inject much more information from external websites into the Twitter discourse. Figure 2 further shows how the dominance of Disbeliever-driven link-sharing behaviors is maintained across every month we observed, ranging from a minimum of $64.64 \%$ of all link-sharing, to a maximum of $87.02 \%$ of all linksharing.

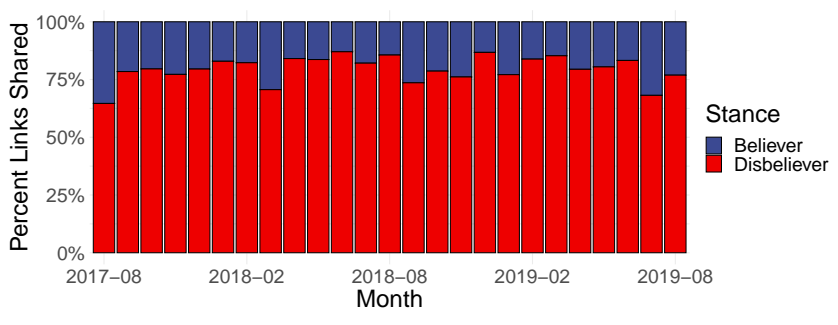

Figure 2: Relative link-sharing behavior of Believers and Disbelievers over time. Disbelievers consistently dominate link-sharing behavior in the two years of online climate change discourse observed in this study.

Nevertheless, moving beyond the sheer volume of link-sharing behaviors, we were also interested in how these behaviors were distributed. Did users share links from the same domains? Figure 3 shows the results of a negative binomial regression model that addresses this question. Here, we show the fitted number of times a link from a specific domain is shared, given the proportion of Disbelievers who share it. 


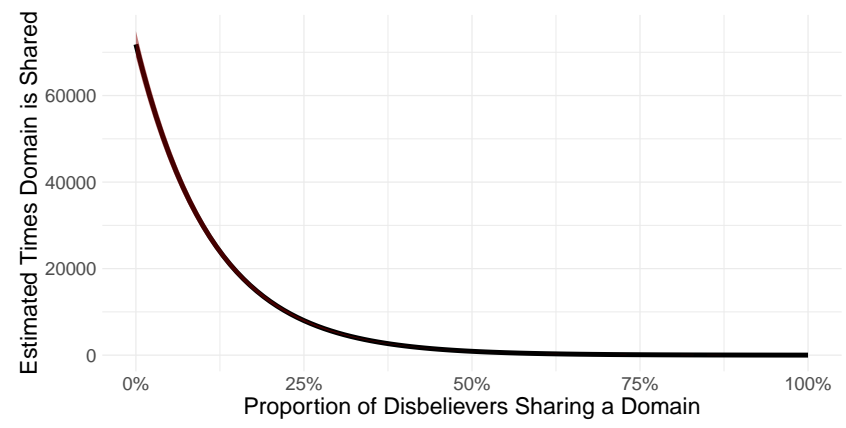

Figure 3: Predictions of a negative binomial regression model, with a $95 \%$ confidence interval. The number of times a link from a certain domain is shared is regressed as a function of the proportion of Disbelievers sharing links from the domain. Links from domains with fewer Disbelievers sharing them tend to be shared more.

Overall, it appears that the more Disbelievers share a link from a specific domain, the less it is shared overall. More specifically, for a given domain $D$, the results of negative binomial regression suggest that every $10 \%$ increment in the proportion of Disbelievers sharing links from domain $D$ predicts a steep $58.46 \%$ decline in the number of times the domain is shared in the entire two-year conversation. Taken together, these findings indicate that Believers tended to share a narrow pool of the most popular domains, while Disbelievers shared a vast range of domains that were ultimately unpopular.

\subsection{How are the information ecosystems of Believers and Disbelievers structured?}

The foregoing analysis tackled link-sharing behavior on an individual level. In this section, we now shift to characterizing the structure of the broader information ecosystems in which these behaviors are embedded. The succeeding analysis operationalizes these questions in terms of domain clusters' features or clusters of domains in monthly domain-by-domain co-sharing networks.

As Figure 4 visualizes, the distribution of stance scores over these domain clusters tends towards the extremes of our stance metric. This indicates that the information ecosystems upon which Believers and Disbelievers draw are largely polarized: Believers share links from a vastly different set of domains than Disbelievers do. However, note also that this polarization is asymmetrical. Whereas Believer domain clusters certainly feature highly positive stance scores $(M=0.8215, S D=0.2739)$, Disbeliever domain clusters display stance scores that are further to the extreme, and much more consistently so $(M=-0.9514, S D=0.1600)$. Intuitively, this tells us that while Disbelievers may also share some domains shared by Believers, many domains shared by Disbelievers are only rarely shared by Believers.

Utilizing network measures, we can sharpen these observations further. From a structural perspective, we detect significant differences in key features of domain clusters associated with Believers and Disbelievers. Figure 5 depicts the mean densities, E/I indices, and sizes of domain clusters obtaining a positive average stance

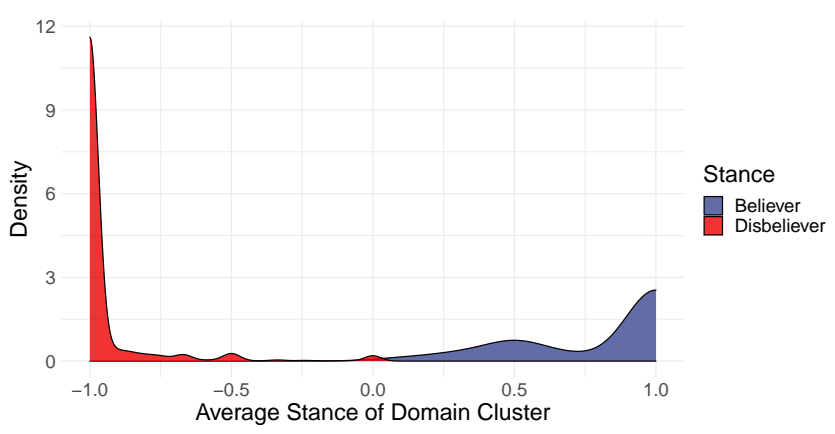

Figure 4: Distribution of stance scores over domain clusters in our dataset. Values tend toward the extremes of the range of possible stance scores, suggesting largely polarized information ecosystems.

score (Believer-leaning clusters) compared to those obtaining negative average stance scores (Disbeliever-leaning clusters).

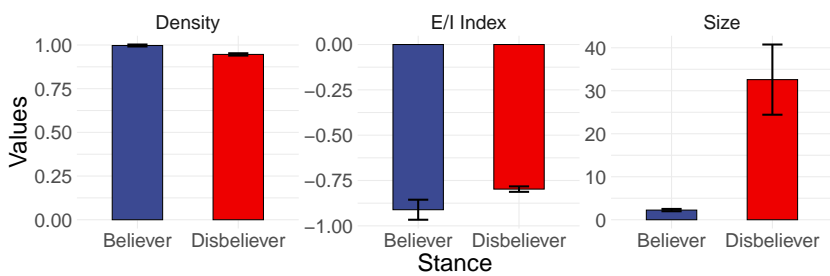

Figure 5: Mean structural features of Believer and Disbeliever domain clusters with $\mathbf{9 5 \%}$ confidence intervals. Statistically significant differences are detected across the three features.

In Figure 5, we see that Believers tend to have denser, more exclusive (more negative E/I indices), and smaller domain clusters than Disbelievers do. We note that, because we are characterizing the features of domain-by-domain networks, these results are not referring to dense, exclusive, and small communities of people. Instead, they pertain to the information resources upon which Believers and Disbelievers draw. Let us unpack each of the findings below.

Because Believers have denser domain clusters than Disbelievers $(t(381)=11.52, p<.001)$, this means that domains tend to be linked to many other domains in their respective domain clusters. Operationally, then, Believers that share links from domain $D_{1}$ in a given cluster are also likely to share links from domain $D_{2}$ in the same cluster. In other words, Believers engage in high levels of domain co-sharing behavior. By contrast, since Disbelievers have slightly less dense domain clusters, the domains they propagate are less likely to be co-shared in this manner.

Now, because Believer domain clusters have more negative E/I indices $(t(79.64)=3.89, p<.001)$, the link-sharing behaviors of Believers also tend to be more exclusive. In other words, domains $D_{1}$ and $D_{2}$ in a Believer domain cluster are less likely to be connected to domain $D_{3}$ in a different cluster. When Believers share links, they therefore tend to do so within a well-defined scope of information 
sources. Disbelievers are more likely to share links from domains belonging to various clusters.

Finally, since Believers have smaller-sized domain clusters, they tend to share links from a more narrow and well-defined range of domains. Larger domain clusters, such as those of Disbelievers, indicate more diverse link-sharing behaviors. In other words, Disbelievers collectively have a more diffuse media diet, whereas Believers stick to a more established set of information sources as a whole.
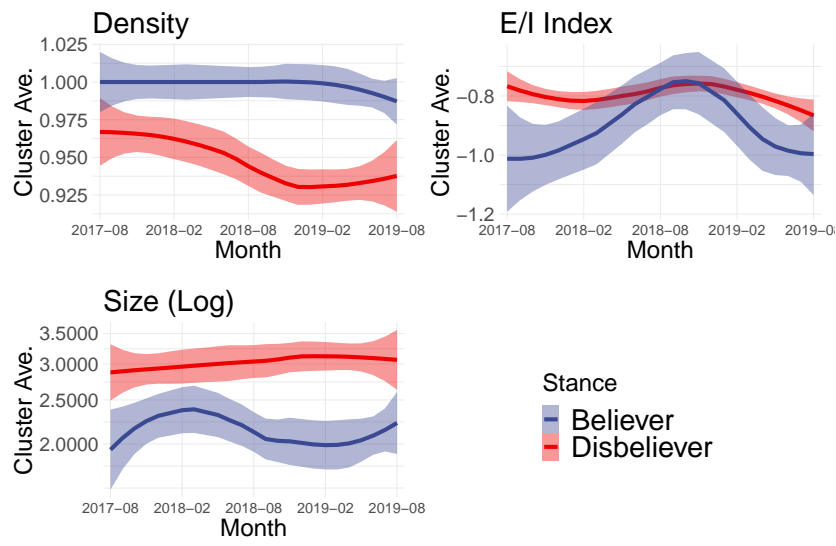

Stance

- Believer

- Disbeliever

Figure 6: Average structural features of Believer and Disbeliever domain clusters over time. Plots depict loess trends with $95 \%$ confidence intervals.

Figure 6 demonstrates that all these structural patterns are relatively consistent across the two years observed. As indicated by the non-overlapping $95 \%$ confidence bands, these differences moreover remain statistically significant $(p<.05)$ for most of the months under consideration. The major exception here pertains to some months in 2018 when the E/I indices of Believer and Disbeliever domain clusters approach nearly identical values. These fluctuations indicate that information ecosystems are thus not static. However, their overall relative patterns nonetheless remain fairly consistent. These findings collectively suggest that differences in the structural features of Believer and Disbeliever information ecosystems are a chronic rather than transient property of the online climate change conversation.

\subsection{What web domains do the information ecosystems of Believers and Disbelievers contain?}

We now present findings on the content of the information ecosystems involved in online climate change discourse. We begin with a high-level, quantitative content analysis focused on the political orientations of known web domains, as well as the salience of known fake news websites. We then present the top 10 web domains with the highest cluster-level centrality for both Believers and Disbelievers.

Figure 7 depicts the empirical cumulative distribution functions (CDFs) of the cluster-level stance scores for domain clusters featuring majority left, right, and center-leaning sources. Pairwise analysis with two-sample Kolmogorov-Smirnov tests indicates statistically significant differences between all CDFs $(p<.001)$. This indicates that Believers and Disbelievers share links from domains of various political orientations at different rates. That said, Disbelievers share majority right sources almost exclusively, followed by majority left sources, then majority center sources.

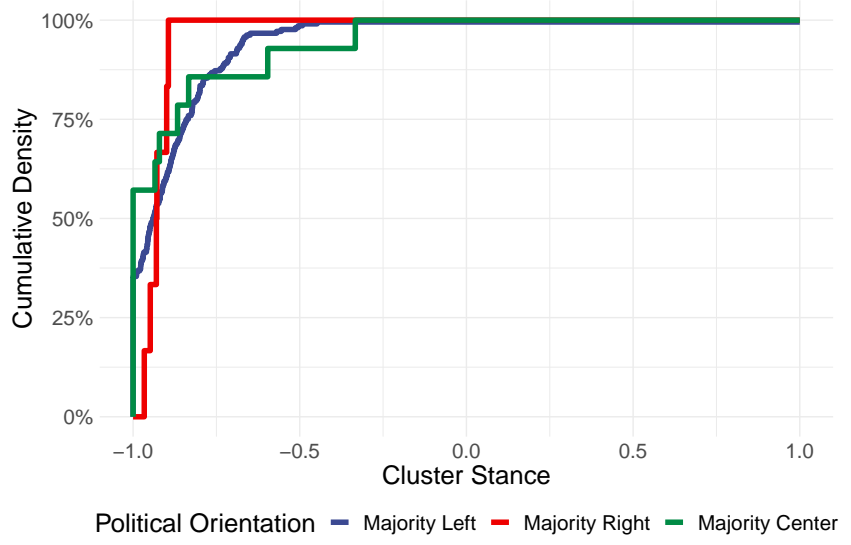

Figure 7: Empirical cumulative distribution functions of average stance of domain clusters which have been classified as majority left, majority right, and majority center.

Next, we focus on fake news sites. We find that Disbelievers are more likely to share links from domains associated with lowcredibility information. Figure 8 shows the CDF of the average stance of domain clusters featuring at least one fake news site in our corpus. Here, we observe that approximately $96.15 \%$ of domain clusters featuring at least one fake news site had an average stance below 0, corresponding to Disbeliever-dominated domain clusters. In the same figure, we also see the distribution of these fake news sites across domain clusters as previously classified by political orientation. The majority of right-leaning domain clusters in the online climate change discussion had the highest proportion of fake news sites.

Finally, to shift from a macro-level analysis to a more specific, qualitative assessment of individual information sources, we examine the domains with the highest cluster centrality over time. Table 1 lists these domains, presenting them in order of highest to lowest cluster centrality values for both Believers and Disbelievers.

Interestingly, several of the most central domains for Believers did not have much to do with climate change explicitly. Domains like instagram are more general-purpose social media, while rollingstone and tvguide have more to do with entertainment and culture. This is a crucial observation, even if it is initially counter-intuitive. This tells us that Believers are disproportionately more likely to incorporate URLs in their tweets which come from websites that also discuss other content, yet may incidentally include climate change content.

However, Believer domains also included more academic sources, like the library website of a university (journalslibunbca), or popular science outlets like scientificamerican and nationalgeographic. Of interest, likewise, is the left-leaning news aggregation site verrit, 

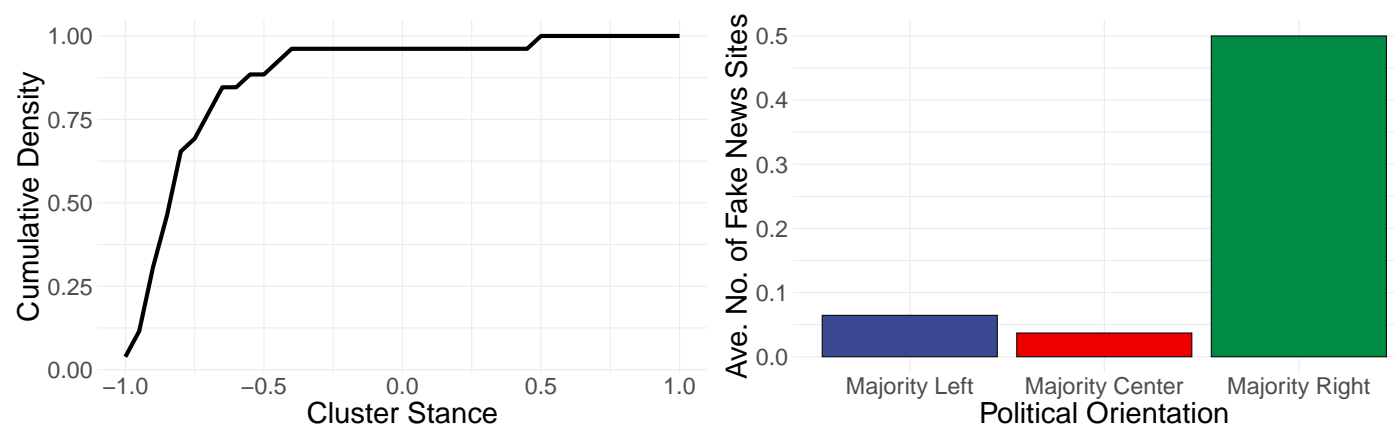

Figure 8: Sharing of fake news sites across domain clusters. Left: Empirical cumulative distribution function of average stance of domain clusters sharing at least one fake news site. Right: Average number of fake news sites shared by domain clusters classified as majority left, majority center, and majority right.

\begin{tabular}{ll}
\hline Believer Domains with Highest Cluster Centrality & Disbeliever Domains with Highest Cluster Centrality \\
\hline $\begin{array}{l}\text { journalslibunbca, tvguide, rollingstone, instagram, thestar, thefader, } \\
\text { verrit, scientificamerican, insightooo, newsnationalgeographic }\end{array}$ & $\begin{array}{l}\text { theguardian, wattsupwiththat, youtube, wrldbg, theblaze, } \\
\text { conservationbytes, committedconservative, sustmememagazine, } \\
\text { environmentguru, climatechangenews }\end{array}$ \\
\hline
\end{tabular}

Table 1: Top 10 domains with the highest average cluster-level centrality for Believers and Disbelievers.

a hub for news that had gained public traction for supporting and being endorsed by Hillary Clinton ${ }^{3}$. This list suggests that while Believer domains may feature scientific content, as well as mainstream social media activity, it may also feature politically charged, liberal-leaning content.

On the other hand, the top domains for Disbelievers strikingly feature explicitly climate-related websites like environmentguru and climatechangenews, in contrast to the more general academic sources found among Believers. This suggests that Disbelievers, despite skepticism about climate change, may still share climate change information, possibly to express doubts or disagreement. Highly conservative sites were also among the most central domains, such as theblaze and the committedconservative, pointing again to a sharp difference to the left-leaning site found among Believers. Finally, we observe that mainstream domains like theguardian and youtube also feature in this list, indicating that not all Disbelievers' information sources are entirely marginal. However, we also note that youtube links may vary widely in terms of information quality, as it may serve as a platform for more in-depth engagement with low-credibility information.

\section{CONCLUSIONS AND FUTURE WORK}

This work examined the information ecosystems of large-scale online climate change discourse over two years. In the context of contentious polarization, our key finding reveals persistent structural and functional asymmetries between the well-consolidated mainstream sources invoked by climate change Believers, versus the more diverse and diffuse fringe sources upon which climate change Disbelievers rely.

\footnotetext{
${ }^{3}$ https://www.washingtonpost.com/news/the-intersect/wp/2017/09/05/what-evenis-verrit-the-news-source-endorsed-by-hillary-clinton/
}

Two insights emerge from this analysis. First, these results based on the bottom-up sharing practices of digital publics strikingly mirror past studies on top-down media representations of climate change $[1,7,33]$. These past studies had suggested that how media institutions represent the issue of climate change has become sharply divided over the years. Our work complements this finding by showing that online accounts on Twitter, whom we computationally associate with climate change Belief and Disbelief, also tend to share information in a bifurcated manner. This suggests that public consumption and propagation of information may echo what information is made available in the wider socio-technical landscape. Moreover, these patterns add an informational dimension to the growing literature on the interactional and affective dimensions of polarization around climate change. [26, 29]. Not only do climate change Believers and Disbelievers appear to interact in isolated and mutually hostile ways, they also seem to rely on divided worlds of information.

Second, while Disbeliever domains remain unpopular throughout the entire social network, their sheer volume points to numerous points of entry into climate change skepticism. Even if Believers share a consistent set of the most popular domains, the fact that Disbelievers may access a wide variety of information sources that may reinforce their views is an important development to consider in conjunction with related concerns over the quality of information in cyberspace [24]. As we emphasize throughout this work, local connections rather than global ones may drive information consumption. Climate change communication efforts and public policy may thus need to attend to these localized network dynamics to adequately respond to low-credibility information, which may undermine legitimate scientific consensus and proliferate conspiracies [13]. That said, the apparent lack of consolidation in information sources in the large-scale data we observe may also 
point to opportunities for policy-makers, as such fringe information ecosystems may potentially still be addressed before more centralized structures develop.

We derived these insights through the design and deployment of a computational methodology integrating machine learning and network science tools [27]. Although the methods employed here tend mainly to employ fundamental social network analysis techniques, they demonstrate how a novel operationalized view of information ecosystems may nonetheless unlock analytical possibilities within the climate change literature, such as the systematic examination of long-term public discourse on a large scale.

However, this framework also constrains the scope of our findings. While we examine observable and quantifiable online behaviors, we do not speak to the internal psychological processes of motivation and cognition underpinning them [18, 32]. Moreover, as computational social scientists have pointed out, the study of Twitter may also not generalize to real-world populations or other social media platforms due to sampling complications [20]. Indeed, while our data collection was not geographically constrained, we limited our analysis to the English language, thereby narrowing our ability to infer cross-cultural conclusions.

In view of these limitations, more complex views of the stances people may hold toward climate change could be integrated toward a non-dichotomous analysis of the dynamics between Believers and Disbelievers. Indeed individual stances toward climate change may be more varied and even dynamic over time, especially given our long-term period of analysis. Temporal analysis could also be extended to domains themselves, to detect whether certain websites become more or less associated with various climate change stances over time.

Future work may thus account for these more complex meaningmaking processes around climate change and other contentious issues when applying our analytical framework. Other research interested in explicit psychological processes may likewise seek to build on our insights through experimental, survey-based, or even ethnographic approaches. Such frameworks would valuably complement the naturalistic analysis we embark on here, by teasing out both quantitative and qualitative nuances of the divide between mainstream and fringe sources. Finally, in accounting for the embeddedness of climate change discourse in the fraught landscape of cyberspace, it may also be beneficial to perform cross-platform analysis and social cybersecurity assessments of potential manipulation through bot activity and information campaigns [6].

\section{ACKNOWLEDGMENTS}

This work was supported in part by the Knight Foundation and the Office of Naval Research (ONR) grants N000141812106 and N000141812108. Additional support was provided by the Center for Computational Analysis of Social and Organizational Systems (CASOS) and the Center for Informed Democracy and Social Cybersecurity (IDeaS). The views and conclusions contained herein are those of the authors and should not be interpreted as representing the official policies, either expressed or implied, of the Knight Foundation, ONR, or the U.S. government.

\section{APPENDIX}

Primary domain names of URLs used to find the political orientation of information clusters as defined in Section 3.4: nbcnews, nypost, usatoday, wsj, cbsnews, abc, univision, huffpost, thehill, vice, politico, businessinsider, buzzfeed, npr, pbs, newsweek, guardian, vox, cnn, washingtonpost, time, bbc, nytimes, msnbc, rushlimbaugh, breitbart, dailycaller, hannity, washingtonexaminer, foxnews.

\section{REFERENCES}

[1] Silke Adam, Ueli Reber, Thomas Häussler, and Hannah Schmid-Petri. 2020. How climate change skeptics (try to) spread their ideas: Using computational methods to assess the resonance among skeptics' and legacy media. PloS One 15, 10 (2020), e0240089.

[2] Jennifer Allen, Baird Howland, Markus Mobius, David Rothschild, and Duncan J Watts. 2020. Evaluating the fake news problem at the scale of the information ecosystem. Science Advances 6, 14 (2020), eaay3539.

[3] Ashley A Anderson and Heidi E Huntington. 2017. Social media, science, and attack discourse: How Twitter discussions of climate change use sarcasm and incivility. Science Communication 39, 5 (2017), 598-620.

[4] Jeremiah Bohr. 2020. Reporting on climate change: A computational analysis of US newspapers and sources of bias, 1997-2017. Global Environmental Change 61 (2020), 102038.

[5] Nicholas Browning and Kaye D Sweetser. 2020. How media diet, partisan frames, candidate traits, and political organization-public relationship communication drive party reputation. Public Relations Review 46, 2 (2020), 101884.

[6] Kathleen M Carley. 2020. Social cybersecurity: An emerging science. Computational and Mathematical Organization Theory 26, 4 (2020), 365-381.

[7] Sedona Chinn, P Sol Hart, and Stuart Soroka. 2020. Politicization and polarization in climate change news content, 1985-2017. Science Communication 42, 1 (2020), $112-129$.

[8] Jessica T Feezell, John K Wagner, and Meredith Conroy. 2021. Exploring the effects of algorithm-driven news sources on political behavior and polarization. Computers in Human Behavior 116 (2021), 106626.

[9] Dana R Fisher, Joseph Waggle, and Philip Leifeld. 2013. Where does political polarization come from? Locating polarization within the US climate change debate. American Behavioral Scientist 57, 1 (2013), 70-92.

[10] R Kelly Garrett, Jacob A Long, and Min Seon Jeong. 2019. From partisan media to misperception: Affective polarization as mediator. Journal of Communication 69, 5 (2019), 490-512.

[11] Binxuan Huang and Kathleen M Carley. 2020. Disinformation and misinformation on Twitter during the novel coronavirus outbreak.

[12] Hank C Jenkins-Smith, Joseph T Ripberger, Carol L Silva, Deven E Carlson, Kuhika Gupta, Nina Carlson, Ani Ter-Mkrtchyan, and Riley E Dunlap. 2020. Partisan asymmetry in temporal stability of climate change beliefs. Nature Climate Change 10, 4 (2020), 322-328.

[13] John T Jost, Eran Halperin, and Kristin Laurin. 2020. Editorial overview: Five observations about tradition and progress in the scientific study of political ideologies. Current Opinion in Behavioral Sciences 34 (2020), iii.

[14] Mark Jurkowitz, Amy Mitchell, Elisa Shearer, and Mason Walker. 2020. US media polarization and the 2020 election: A nation divided.

[15] David Krackhardt and Robert N Stern. 1988. Informal networks and organizational crises: An experimental simulation. Social Psychology Quarterly 51, 2 (1988), 123-140.

[16] Sumeet Kumar. 2020. Social media analytics for stance mining: A multi-modal approach with weak supervision. Ph.D. Dissertation. Carnegie Mellon University.

[17] Thomas J Leeper. 2014. The informational basis for mass polarization. Public Opinion Quarterly 78, 1 (2014), 27-46.

[18] Stephan Lewandowsky. 2020. Climate change, disinformation, and how to combat it. Annual Review of Public Health 42 (2020), 1-21.

[19] Renée Moernaut, Jelle Mast, Martina Temmerman, and Marcel Broersma. 2020. Hot weather, hot topic. Polarization and sceptical framing in the climate debate on Twitter. Information, Communication \& Society 0, 0 (2020), 1-20.

[20] Fred Morstatter, Jürgen Pfeffer, Huan Liu, and Kathleen Carley. 2013. Is the Sample Good Enough? Comparing Data from Twitter's Streaming API with Twitter's Firehose. In Proceedings of the International AAAI Conference on Web and Social Media, Vol. 7. AAAI, Cambridge, MA, USA, 400-408.

[21] Warren Pearce, Kim Holmberg, Iina Hellsten, and Brigitte Nerlich. 2014. Climate change on Twitter: Topics, communities and conversations about the 2013 IPCC Working Group 1 report. PloS One 9, 4 (2014), e94785.

[22] Cass R. Sunstein. 2007. Republic.com 2.0. Princeton University Press, Princeton, NJ. http://www.jstor.org/stable/j.ctt7tbsw

[23] Vincent A Traag, Ludo Waltman, and Nees Jan van Eck. 2019. From Louvain to Leiden: Guaranteeing well-connected communities. Scientific Reports 9, 1 (2019), $1-12$. 
[24] Aman Tyagi. 2021. Challenges in Climate Change Communication on Social Media Ph.D. Dissertation. Carnegie Mellon University.

[25] Aman Tyagi, Matthew Babcock, Kathleen M Carley, and Douglas C Sicker. 2020. Polarizing tweets on climate change. In International Conference on Social Computing, Behavioral-Cultural Modeling and Prediction and Behavior Representation in Modeling and Simulation. Springer, Virtual Conference, 107-117.

[26] Aman Tyagi, Joshua Uyheng, and Kathleen M Carley. 2020. Affective polarization in online climate change discourse on Twitter. In International Conference on Advances in Social Network Analysis and Mining. IEEE/ACM, Virtual Conference, 443-447.

[27] Joshua Uyheng, Thomas Magelinski, Ramon Villa-Cox, Christine Sowa, and Kathleen M Carley. 2020. Interoperable pipelines for social cyber-security: Assessing Twitter information operations during NATO Trident Juncture 2018 Computational and Mathematical Organization Theory 26, 4 (2020), 465-483.

[28] Sebastián Valenzuela, Ingrid Bachmann, and Matías Bargsted. 2019. The personal is the political? What do Whatsapp users share and how it matters for news knowledge, polarization and participation in Chile. Digital fournalism 9, 2 (2019)
$1-21$.

[29] Christel W van Eck, Bob C Mulder, and Sander van der Linden. 2020. Echo chamber effects in the climate change blogosphere. Environmental Communication 15, 2 (2020), 1-8.

[30] Hong Tien Vu, Matthew Blomberg, Hyunjin Seo, Yuchen Liu, Fatemeh Shayesteh, and Hung Viet Do. 2020. Social media and environmental activism: Framing climate change on Facebook by global NGOs. Science Communication 43, 1 (2020), 91-115.

[31] Anne E Wilson, Victoria Parker, and Matthew Feinberg. 2020. Polarization in the contemporary political and media landscape. Current Opinion in Behavioral Sciences 34 (2020), 223-228.

[32] Gabrielle Wong-Parodi and Irina Feygina. 2020. Understanding and countering the motivated roots of climate change denial. Current Opinion in Environmental Sustainability 42 (2020), 60-64.

[33] Dannagal G Young and Katherine Anderson. 2017. Media diet homogeneity in a fragmented media landscape. Atlantic fournal of Communication 25, 1 (2017), $33-47$ 